DOE /ER/ $13326--T 3$

DE92 007813

\title{
DISCLAIMER
}

This report was prepared as an account of work sponsored by an agency of the United States Government. Neither the United States Government nor any agency thereof, nor any of their employess, makes any warranty, express or implied, or assumes any legal liability or responsibility for the ascuracy, completeness, or usefulness of any information, apparatus, product, or process disclosed, or represents that its use would not infringe privately owned rights. Reference herein to any specific commercial product, process, or service by trade naine, trademark, manufacturer, or otherwise does not necessarily constitute or imply its endorsement, recommendation, or favoring by the United States Government or any agency thereof. The views and opinions of authors expressed herein do not necessarily state or reflect those of the United States Government or any wgency thereof.

\section{Research Briefing on \\ Selected Opportunities in Atomic, Molecular, and Optical Sciences}

$$
\text { FGO5.8SER } 13326
$$

\section{Committee on Atomic, Molecular, and Optical Sciences}

Boatrd on Physics and Astronomy

Commission on Physical Sciences, Mathematics, and Applications

National Research Council

National Academy Press

Washington, D.C. 1991 
NOTICE: The project that is the subject of this report was approved by the Governing Board of the National Research Council, whose members are drawn from the councils of the National Academy of Sciences, the National Academy of Engineering, and the Institute of Medicine. The members of the committee responsible for the report were chosen for their special competences and with regard for appropriate balance.

This report has been reviewed by a group other than the authors according to procedures approved by a Report Review Committee consisting of members of the National Academy of Sciences, the National Academy of Engineering, and the Institute of Medicine.

The National Academy of Sciences is a private, nonprofit, self-perpetuating society of distinguished scholars engaged in scientific and engineering research, dedicated to the furtherance of science and technology and to their use for the general welfare. Upon the authority of the charter granted to it by the Congress in 1863, the Academy has a mandate that requires it to advise the federal government on scientific and technical matters. Dr. Frank Press is president of the National Academy of Sciences.

The National Academy of Engineering was established in 1964, under the charter of the National Academy of Sciences, as a parallel organization of outstanding engineers. It is autonomous in its administration and in the selection of its members, sharing with the National Academy of Sciences the responsibility for advising the federal government. The National Academy of Engineering also sponsors engineering programs aimed at meeting national needs, encourages education and research, and recognizes the superior achievements of engineers. Dr. Robert $M$. White is president of the National Academy of Engineerıng.

The Institute of Medicine was established in 1970 by the National /scademy of Sciences to secure the services of eminent members of appropriate professions in the examination cf policy matters pertaining to the health of the public. The Institute acts under the responsibility given to the National Academy of Sciences by its congressional charter to be an adviser to the federal government and, upon its own initiative, to identify issues of medical care, research, and education. Dr. Samuel $O$. Thier is president of the Institute of Medicine.

The National Research Council was established by the National Academy of Sciences in 1916 to associate the broad community of science and technology with the Academy's purposes of furthering knowledge and of advising the federal government. Functioning in accordance with general policies determined by the Academy, the Council has become the principal operating agency of both the National Academy of Sciences and the National Academy of Engineering in providing services to the government, the public, and the scientific and engineering communities. The Council is administered jointly by both Academies and the Institute of Medicine. Dr. Frank Press and Dr. Robert M. White are chairman and vice chairman, respectively, of the National Research Council.

This project was supported by the Department of Energy under Grant No. DE-FG05-85ER13326 and by the National Science Foundation under Grant No. PHY-8921799.

Additional copies of this document are available from:

Board on Physics and Astronomy

HA 562

National Research Council

2101 Constitution Avenue, NW

Washington, DC 20418

Printed in the United States of America 


\section{Committee on Atomic, Molecular, and Optical Sciences}

GORDON H. DUNN, Joint Institute for Laboratory Astrophysics, Chair MICHAEL LUBELL, City College, CUNY, Past Chair

JOHN E. BJORKHOLM, AT\&T Bell Laboratories DANIEL GRISCHKOWSKY, IBM T.J. Watson Research Center ANDREW U. HAZI, Lawrence Livermore Laboratory WILLIAM KLEMPERER, Harvard University

PETER KOCH, State University of New York, Stony Brook

DANIEL J. LARSON, University of Virginia

DONALD H. LEVY, University of Chicago

WILLIAM H. MILLER, University of California, Berkeley

RONALD PHANEUF, Oak Ridge National Laboratory

DAVID E. PRITCHARD, Massachusetts Institute of Technology

ROBERTA SAXON, SRI International

RICHARD E. SMALLEY, Rice University

Ronald D. Taylor, Senior Program Officer 


\section{Board on Physics and Astronomy}

FRANK D. DRAKE, University of California, Santa Cruz, Chair LLOYD ARMSTRONG, Johns Hopkins University

W. DAVID ARNETT, University of Arizona

HOWARD C. BERG, Harvard University

R. STEPHEN BERRY, University of Chicago

WILLIAM F. BRINKMAN, AT\&T Bell Laboratories

GEORGE W. CLARK, Massachusetts Institute of Technology

HAROLD P. FURTH, Princeton University

MARTHA P. HAYNES, Cornell University

CHARLES F. KENNEL, University of California, Los Anģeles

WALTER KOHN, University of California, San Diego

STEVEN E. KOONIN, California Institute of Technology

LEON LEDERMAN, Fermi National Accelerator Laboratory

VERA RUBIN, Carnegie Institution of Washington

DAVID N. SCHRAMM, University of Chicago

DANIEL TSUI, Princeton University

STEVEN WEINBERG, University of Texas

Donald C. Shapero, Staff Director

Robert L. Riemer, Associate Staff Director

Ronald D. Taylor, Senior Program Officer

Susan M. Wyatt, Administrative Associate

Mary Riendeau, Administrative Secretary

Anne K. Simmons, Secretary 


\section{Commission on Physical Sciences, Mathematics, and Applications}

NORMAN HACKERMAN, Robert A. Welch Foundation, Chairman

PETER J. BICKEL, University of California, Berkeley

GEORGE F. CARRIER, Harvard University

HERBERT D. DOAN, The Dow Chemical Company (retired)

DEAN E. EASTMAN, IBM T.J. Watson Research Center

MARYE ANNE FOX, University of Texas

PHILLIP A. GRIFFITHS, Duke University

NEAL F. LANE, Rice University

ROBERT W. LUCKY, AT\&T Bell Laboratories

CHRISTOPHER F. MCKEE, University of California, Berkeley

RICHARD S. NICHOLSON, American Association for the Advancement of Science

JEREMIAH P. OSTRIKER, Princeton University Observatory

ALAN SCHRIESHEIM, Argonne National Laboratory

ROY F. SCHWITTERS, Superconducting Super Collider Laboratory

KENNETH G. WILSON, Ohio State University

NORMAN METZGER, Executive Director 


\section{Preface}

The Committee on Atomic, Molecular, and Optical Sciences of the National Research Council's Board on Physics and Astronomy has prepared this research briefing at the request of the director of the Office of Energy Research of the Department of Energy (DOE) to provide the DOE, other federal agencies, and the atomic, molecular, and optical (AMO) sciences community at large with a rapid assessment of some forefront research areas. The request was motivated by a concern for the health of AMO sciences mutually shared by DOE, the National Research Council's Board on Physics and Astronomy, and the AMO community.

In addition to giving a glimpse of the nature and character of the AMO field, this research briefing identifies and discusses a few selected opportunities and highlights from forefront research in the field, and it acknowledges specific issues associated with these areas of opportunity. No attempt has been made to conduct a detailed assessment of the field or to be comprehensive in the perspective given. Therefore, no attempt has been made to develop recommendations or set programmatic priorities.

Some reading this briefing, such as program officers or young researchers, may do so with programmatic or research priorities in mind, and it is important to set a context for that. The broad diversity of the field makes the setting of priorities extremely complex compared with that for some more sharply focused disciplines such as high-energy physics. The Committee on Atomic, Molecular, and Optical Sciences has recently proposed that a detailed study of the scientific and technological opportunities of the field be conducted by the National Research Council. The broader study will assess the state of knowledge of the field of atomic, molecular, and optical sciences and identify the priorities of the community in pursuing its research agenda over the next several years. The latter task will be done from a variety of perspectives, e.g., impact on testing and understanding fundamental laws and impact on energy technologies, other sciences, the environment, commerce, and so forth. It is expected that this broader study will not only assist federal agencies in future program planning, but will also enable those researchers who are just entering the field to identify those areas that show special promise for addressing the nation's needs. The proposed study has received the endorsement of the Department of Energy.

Although the examples of research opportunities discussed in this briefing were not chosen on the basis of a perceived relation to missions of the Department of Energy, it can be argued that the AMO sciences are more broadly integrated into the department's missions than is any other single discipline. There are thus well-established impacts in fusion, fission, fossil, and solar energy production; clear needs from the field for studying impacts of deposition of energy in the environment, whether from weapons or energy production and dissemination; and contributions in areas of energy conservation, such as the development of efficient lighting. Indeed, it should be pointed out that some areas with little or no mention in this briefing are among those that have the greatest programmatic impact on energy technologies. An example of this is the reactive scattering of electrons by molecules, processes that are extremely important in plasma deposition of solar cells, edge plasmas of tokamaks, and evolution of the earth's atmosphere.

Since this research briefing was initiated on the basis of concern for the health of the AMO field, the committee believes that it is appropriate to comment briefly on the support structure and trends for the field. Support is not discussed elsewhere in this research briefing. Just as the field itself is diverse in character, so also is funding for the field. Core funding to university-based AMO sciences comes primarily through the National Science Foundation, the Department of Energy, the Department of Defense, and the National Aeronautics and Space Administration. Strong programs also exist at national and industrial laboratories, with funding through some of the same agencies and, of course, private companies. Over the past five years, the modest increases in university funding for AMO research that have been realized have not kept up with the cost of doing forefront research. This has resulted in the decline during that period in the ability of the AMO community to do forefront research. This situation is not very different from that experienced in other fields of science over the same time frame; however, much more serious 
is the fact that opportunities in some segments (for example, optical physics) of the field have virtually exploded over the same period. As program managers have tried to respond to these opportunities as best they could, funding for essentially all parts of the field has thinned dangerously. The diversity of the field and the fact that research in much of the field is basic to the development of advanced technologies have led to a structure wherein much of the funding is tied to relevant technologies.

The Committee on Atomic, Molecular, and Optical Sciences is grateful to the reviewers for providing feedback on short notice. The committee also acknowledges and thanks J.B. Delos, R.W. Falcone, T.F. Gallagher, D. Herschbach, R.E. Slusher, A.F. Starace, G.I. Stegeman, R.H. Stolen, C. Wieman, and D.J. Wineland for their useful contributions to the report. Finally, the committee appreciates the interest expressed by the Office of Energy Research in laying down the challenge to the coinmunity of preparing this research briefing. 


\section{Contents}

1 INTRODUCTION $\ldots \ldots \ldots \ldots \ldots \ldots \ldots \ldots \ldots \ldots \ldots \ldots \ldots \ldots \ldots \ldots$

II SELECTED RESEARCH HIGHLIGHTS IN AMO SCIENCES $\ldots \ldots \ldots \ldots \ldots \ldots \ldots \ldots \ldots$

The Laser-Atom Revolution . . . . . . . . . . . . . . . . . . . . . . 2

Fundamental Laws and Symmetries . . . . . . . . . . . . . . . . . 2

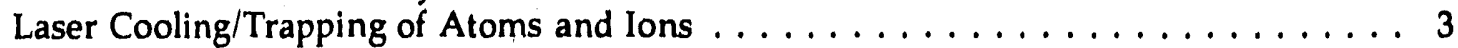

Atoms in Strong Fields $\ldots \ldots \ldots \ldots \ldots \ldots \ldots \ldots \ldots \ldots$

Chaos ................................ 4

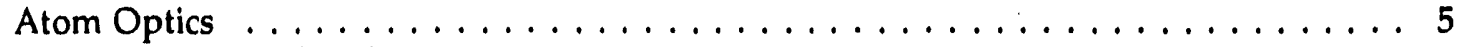

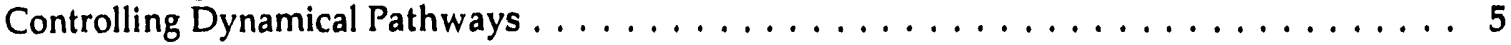

Reactions with Aligned Reactants $\ldots \ldots \ldots \ldots \ldots \ldots \ldots \ldots \ldots$

Preparation of Transient Configurations and the Transition State $\ldots \ldots \ldots \ldots \ldots 6$

Altering Dynamical Pathways by Intermolecular Interactions $\ldots \ldots \ldots \ldots \ldots \ldots$

Nonclassical States of Light $\ldots \ldots \ldots \ldots \ldots \ldots \ldots \ldots \ldots \ldots \ldots$

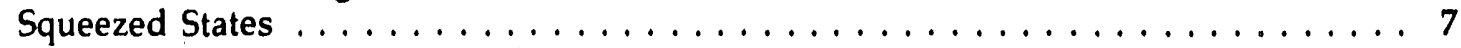

Quantum Twinning . . . . . . . . . . . . . . . . . . . 8

Number States . . . . . . . . . . . . . . . . . . . . . . . 8

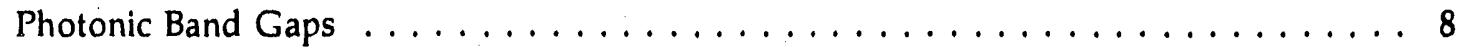

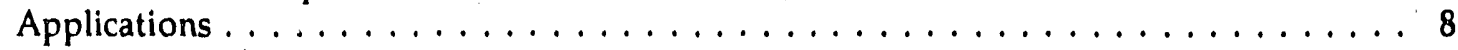

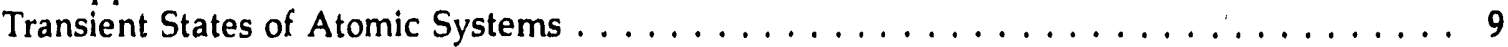

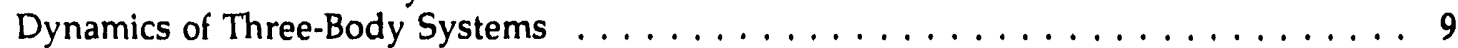

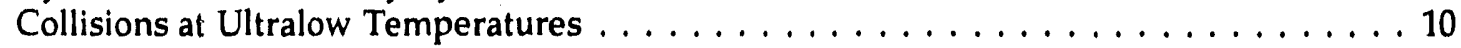

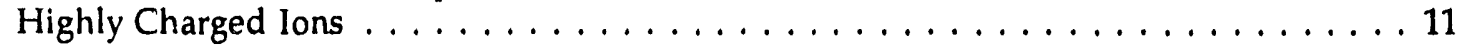

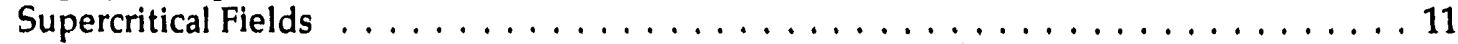

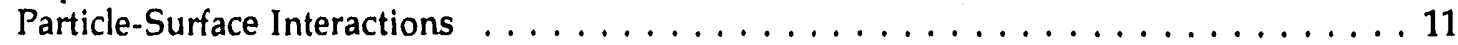

New Light Generation and Handling . . . . . . . . . . . . . . . . . . . . . . . 12

Optically Generated Beams of Sub-picosecond Pulses of $\mathrm{THz}$ Radiation . . . . . . . 12

Second Harmonic Generation in Guided Wave Structures . . . . . . . . . . . . . . 13

Coherent XUV Radiation from Strong-Field Multiphoton Processes . . . . . . . . . . 14

Ultrafast X-ray Pulses from Laser-Produced Plasmas . . . . . . . . . . . . . 15

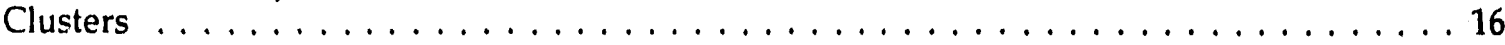

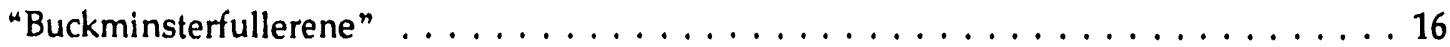

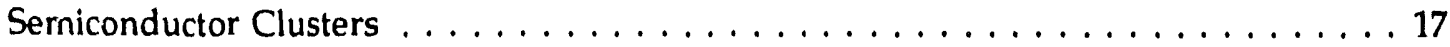

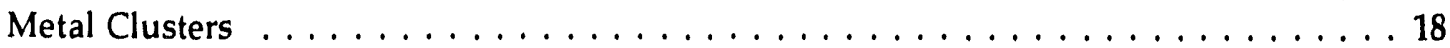

III ATOMIC PHYSICS AT USER FACILITIES $\ldots \ldots \ldots \ldots \ldots \ldots \ldots \ldots \ldots \ldots \ldots$

Synchrotron Radiation Facilities $\ldots \ldots \ldots \ldots \ldots \ldots \ldots \ldots \ldots$

Particle Accelerator Facilities . . . . . . . . . . . . . . . . . . . . . . . . 19

Heavy-Ion Storage-Ring Facilities . . . . . . . . . . . . . . . . . . . . . . 19

IV IMPACTS OF AMO SCIENCES ON MODERN TECHNOLOGIES $\ldots \ldots \ldots \ldots \ldots$ 


\section{I \\ Introduction}

Excitement and advances in the atomic, molecular, and optical (AMO) sciences seem to be approaching a zenith. Recent achievements were seen only in broad generality just five years ago when Physics Through the 1990s (the "Brinkman Report"; National Academy Press, Washington, D.C., 1986) was issued. The AMO sciences, which gave birth to the laser, have developed this tool to yield scientific and technological advances that once could only be dreamed of. Other innovative technologies such as trapping and cooling of neutral atoms and ions, pulse valves for supersonic jets to facilitate cluster investigations, and the continued development of large, ultrafast computers and technologies making highly charged ions available for study have likewise made this a time of exciting progress and scientific opportunity. Recently, we have witnessed some profound developments:

- AMO sciences and high-energy particle physics have combined efforts to deduce coupling constants in the standard model of electroweak interactions.

- Particles have been cooled to record temperatures of about 1 microkelvin.

- Quantum fluctuations of light have been reduced in "squeezed states."

- Real-time "sriapshots" of molecular dynamics can now be made.

These and other developments and opportunities are discussed in a context intended to familiarize the reader a little with the nature of the AMO scitnces. The field and its goals and foci are intensely diverse, with practitioners in physics, chemistry, astrophysics, aeronomy, plasma physics, and other areas. The world's most refined and accurate measurements occur in the AMO sciences, largely brought about by the fact that time and frequency are the most accurately measurable physical quantities, and these fall primarily in the AMO domain. Thus, there is a commensurate impact on the testing and understanding of fundamental laws of nature. At the same time, since the energies involved are those characteristic of our everyday lives, the AMO sciences intimately affect a broad spectrum of technologies. Other science areas are tightly coupled to the field, often capitalizing on analytical techniques or instrumentation that originated in AMO. The impact and diversity of AMO physics are in some measure evidenced by its record of literature cited, which leads all fields of science with more than 90 percent of its publications referenced.

The Divisions of Atomic, Molecular, and Optical Physics and of Chemical Physics in the American Physical Society, when taken together, make up the second largest group in the society, second only to the Division of Condensed Matter Physics. There are, in addition, many practitioners affiliated only with the Laser Science Topical Group, the American Chemical Society, or the Optical Society of America. Work in the field is traditionally carried out as "small science" involving individual investigators and small groups of students or colleagues. National facilities are used when approprite, and again the research is usually carried out by small groups that employ the facilities on a "user" basis.

The AMO sciences play a major role in education. Approximately 18 percent of the nation's PhDs in physics come from AMO. Students educated in this "small science" are well prepared as experts not only in their scientific specialties, but also in many modern technologies such as lasers, ultrahigh vacuums, fast electronics, computers, and cryczenics. Graduates educated in the field are in demand. In a recent survey (Physics Faculty Workforn. Study, Committee on Opportunities in Physics, American Institute of Physics, 1991) qualified graduates in condensed matter physics and AMO were found to be in short supply relative to the demand as measured by open faculty positions.

This research briefing has been assembled 
rapidly, without the give-and-take needed to assess priorities. Thus the inclusion of some topics here and the absence of others should not be construed as representing an assessment of relative importance. Rather, this briefing represents a sampling of a few of the many important opportunities in each of the three areas of the AMO sciences-atomic, molecular, and optical-where exciting progress has recently occurred and where increased efforts in the coming few years should produce important new results.

In the discussion and examples that follow, no distinction is generally made between theoretical or experimental work. One of the very strong features of the AMO field is that theory and experiment generally march very closely together. For both, the challenges are severe and the opportunities great. For example, the understanding of nonlinear dynamics is looked upon as one of the great challenges of our time, and both theoretical and experimental $u$ ork in AMO are recognized as fertile proving grounds in the active field of chaos discussed in this briefing.

\section{II

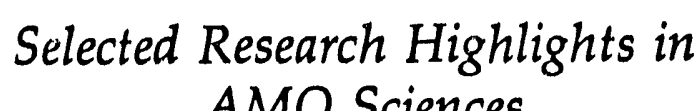 AMO Sciences}

\section{THE LASER-ATOM REVOLUTION \\ Fundamental Laws and Symmetries}

One of the unique aspects of atomic physics is that a variety of quantities can be measured with extremely high precision. In many cases this precision can be used to probe very fundamental laws of physics. Going beyond qua:1tum electrodynamics, which, thanks to atomic physics measurements, is now the most precisely tested and confirmed theory in physics, there have been recent advances in addressing a number of other fundamental physical questions. Much of this work is possible because of developments in atomic and laser technology, which now make it possible, for example, to measure atomic energy level splittings to tiny fractions of a hertz ( Hz). Such a capability has been used to test whether these splittings are sensitive to a variety of parameters that address basic questions of physics. For example, demonstrations that the splittings are independent of the spatial orientation of the apparatus have provided much better confirmation of the isotropy of space. Careful measurements of how the splittings change with atomic velocity and distance from the earth have provided precise confirmation of other aspects of the special and general theories of relativity. Precision frequency measurements have also been used to make very sensitive tests for the existence of possible nonlinear terms in basic equations of quantum mechanics.

A shift in the energy splittings between nondegenerate states of atoms or molecules that depends linearly on a dc electric field would be an indication of a time-reversal ( $\mathrm{T}$ )-violating electric dipole moment. In the past few years, advances in laser and atomic beam technology have facilitated improvements in the search for such dipole moments. These experiments now provide the best limits on T-violating interactions between the electrons and the nuclei and rule out a number of elementary particle theories that have been suggested. In another examination of a fundamental symmetry, the precise comparison of the masses of protons and antiprotons that are held in an ion trap has led to a dramatically improved test of the CPT theorem for baryons which says that any Lorentz-invariant field theory must be invariant under the combined operations of charge conjugation $C$, parity inversion $P$, and time reversal $T$.

The nonconservation of parity in atoms has also been an area of recent progress. Precise measurements of this quantity, combined with dramatically improved calculations of the structure of heavy atoms and the measured masses of the $Z^{\circ}$ and $W^{ \pm}$, now provide the most accurate test of the renormalizability of the standard model of electroweak unification. Precision atornic physics measurements of parity 
nonconservation also provide the potential for investigating extensions of the Standard Model that contain extra $Z^{\circ}$ bosons.

\section{Laser Cooling/Trapping of Atoms and Ions}

Record low particle temperatures have recently been achieved using laser techniques. Atoms and ions are now routinely "lasercooled" in many laboratories throughout the world. Cooling is accomplished by three basic methods, which use damping from radiation pressure ("Doppler cooling"), deceleration from optical dipole forces ("polarization gradient" or "Sisyphus" cooling), or optical pumping into low kinetic energy staires that interact only very weakly with the pumping source ("population trapping" cooling or "sideband cooling" in the resolved sideband limit (for trapped particles)). Temperatures near 1 microkelvin are now obtained. By various combinations of electromagnetic fields, including laser fields, atoms or ions can be trapped for long periods of time (>1 $\mathrm{min}$ ), which allows close examination of various properties. The techniques can be applied to a number of areas, including highprecision spectroscopy, low-temperature collision studies, atom manipulation, the production and study of unusual condensed phases of matter, and the creation of the most accurate clocks ever devised.

In spectroscopic studies, laser cooling and trapping allow very long interrogation times and significantly reduced Doppler shifts. This can lead to very-high-resolution, high-accuracy spectroscopy. Applications include atomic clocks and tests of basic symmetries and interactions. "Atomic fountains" with interaction times of about $1 \mathrm{sec}$ have now been realized. This will yield frequency standards with resolution much better than that of any previous atomic clocks. Linewidths of atomic transitions in ions have been measured to be less than $0.001 \mathrm{~Hz}$ in the radio-frequency spectrum and less than $100 \mathrm{~Hz}$ in the optical spectrum. Ultimate accuracies are expected eventually to exceed 1 part in $10^{18}$. Accurate clocks are used in precise navigation, for very long baseline interferometry, and for tests of general relativity.
Tests of local Lorentz invariance, tests of the linearity of quantum mechanics, and searches ror anomalous long-range interactions have already been maue. Higher-accuracy searches for time-reversal violation through detection of electric dipole moments in atonis are planned. Experiments that are currently in progress should substantially improve our knowledge of the electron-proton charge equality as well as parity nonconservation effects associated with neutral weak currents. The emphasis of this latter work is on the trapping of short-lived radioactive isotopes using light pressure, which will also allow new fundamental studies of beta decay.

Laser cooling makes possible atomic collision studies at extremely low energies. At very low velocities where the atom's de Broglie wavelength is long compared to the range of the interaction potentials, scattering becomes a distinctly quantum mechanical phenomenon. In addition, the scattering can be dominated by weak, long-range forces, and in the case where the de Broglie wavelength of the atom is large compared to the attractive region near a material surface, the atom may experience only the repulsive part of the surface interaction, thus bouncing elastically off the surface rather than sticking to it. This may help provide nearly ideal atom "boxes."

Optical forces have been used to slow neutral atoms, steer atomic beams, and make atom "traps." Applications of atom traps may include storage and manipulation of atomic antimatter, which must not come into contact with ordinary matter to avoid annihilation. Laser cooling should also provide cold, high-luminosity beams in high-energy ion storage rings.

Collections of atomic ions contained in ion traps can be viewed as plasmas. At the very low temperature provided by laser cooling, the plasmas exhibit liquid and solid properties. For neutral atoms that interact weakly as bosons, such as hydrogen or the alkalis, different atoms can have the same spatial wave function. If a sample of such atoms is trapped and sufficiently cooled, it may be possibie to observe BoseEinstein condensation. 


\section{Atoms in Strong Fields}

Recent experimental and technological advances provide dramatic new opportunities for the study of atomic systems in strong static and oscillatory fields. These studies take us into regions where entirely new phenomena may occur and where appropriate theoretical descriptions have not been developed. Early theoretical and experimental studies considered only weak radiation fields, fields weak enough to be treated as small perturbations to the atomic fields. For single-photon processes, which can be observed with classical sources, and for loworder multiphoton processes, perturbation approaches are perfectly adequate. However, for high-order multiphoton processes, perturbation approaches are of little utility.

The issue of high-order processes is far from academic, as recent developments in laser technology have made them readily accessible. A few examples serve to illustrate this point. Twelve-photon ionization of $\mathrm{Xe}$ atoms by $1-\mu \mathrm{m}$ radiation and 100-photon ionization of Xe by 10 $\mu \mathrm{m}$ radiation are both readily observed. When $\mathrm{Xe}$ atoms are exposed to light of even greater intensity, it is possible to remove not only one electron, but also the entire outer shell, a process requiring the investment of $600 \mathrm{eV}$ in the atom, i.e., hundreds of photons. Similarly, wher the Xe atom is exposed to intense fields, an electron ejected from the atom by the radiation field can acquire large amounts of kinetic energy, a surprising observation because free electrons cannot absorb energy. The interaction of molecules with intense radiation fields offers an even richer variety of outcomes, since the possibility of dissociation is added to ionization. Today it is possible to produce fields as strong as the field binding the electron in the ground state of hydrogen and fields where the ponderomotive or "jiggle" energy of electrons is within an order of magnitude of the rest energy. More surprising results seem likely.

The recent experimental advances have posed a formidable task for the theorist. The essential question is how to think about the interaction of atoms with intense radiation fields. One way of phrasing the question is to ask how one correctly departs from the previous perturbation theory picture of radiation fields to the description of arbitrarily strong fields.

At the moment there is a vigorous experimental effort focused on the study of atoms and molecules in strong laser fields of about $10^{9}$ $\mathrm{V} / \mathrm{cm}$, and on the interaction of highly excited atoms, in which the valence electron is only weakly bound, with much more modest microwave fields of about $10^{4} \mathrm{~V} / \mathrm{cm}$. Theoretical efforts parallel the experimental activity, and the issue of what is really at the root of the experimental observations remains a source of lively debate.

\section{Atomic systems have become one of the} primary testing grounds of how new concepts in nonlinear dynamics and chaos apply to nondissipative microscopic systems. Conversely, developments in the theory of chaotic dynamics provide opportunities for interpretation of complex phenomena that previously were ignored or not understood. "Chaos" refers to a system that follows deterministic laws, but whose trajectories are so (exponentially) unstable that longterm prediction is impossible. Almost all macroscopic, classical systems, from billiard balls colliding without friction on a table to charged particles moving under electromagnetic forces, may undergo chaotic motion. However, application of the linear, time-dependent Schroedinger equation to bounded quantal systems yields quasiperiodic solutions that do not behave chaotically. (For open systems, less is known formally.) "Quantum chaology," the study of quantal systems whose classical counterparts exhibit chaos, seeks to reveal whether and how quantal systems may approach a classically chaotic limit. This has opened a new domain of study of the correspondence principles introduced by Bohr and others.

Chemical cycles, vibrations in polyatomic molecules and clusters, bistability in lasers, and mechanical motions of two or more ions in traps all manifest chaotic classical dynamics in different ways. The behavior of the density of states and the statistical properties of quantal spectra 
in, e.g., atoms and molecules (and nuclei, as well) also seem to reflect the presence or absence of chaos in the corresponding classical system. Two microscopic atomic systems, however, have become the complementary, principal testing grounds of quantum chaology in simple, real physical systems. One is the time-independent problem of the photoabsorption spectrum of atoms in a magnetic field that exerts a force rivaling the Coulomb force binding the electron and core ion in the final state. The other is the time-dependent problem of the excitation and ionization of highly excited hydrogen or other Rydberg atoms by strong microwave electric fields. For hydrogen atoms, both these problems exhibit chaos in their nonlinear, classical dynamics. The surprise and excitement have been that various fingerprints of the chaotic behavior actually show up in real experimental data.

\section{Atom Optics}

Ever since the invention of the atomic beam, physicists have sought techniques for handling beams of neutral atoms comparable to the optical methods routinely used for beams of photons and charged particles. Several recent experiments represent large steps in this direction and herald the beginnings of "atom optics."

A carefully polished quartz hemisphere was positioned so that its center coincided with a hole in a cell of cold (less than $0.5 \mathrm{~K}$ ) hydrogen atoms. When the quartz hemisphere was coated with a liquid $\mathrm{He}$ film and cooled, it became an excellent atom reflector, reflecting the $\mathrm{H}$ atoms leaving the hole back into it, thereby reducing the rate of loss of $\mathrm{H}$ from the bottle. At the lowest surface temperature studied $(0.1 \mathrm{~K})$, the loss rate was reduced by a factor exceeding 4 , indicating that the mirror ieflected at least 75 percent of the incident atoms back into the hole. An atom mirror for room-temperature atoms was also recently made from small gold crystals.

The second development is a transmission diffraction grating. This grating consists of a series of slots formed by a rcsw of free-standing small bars, each $1000 \AA$ wide with a center-tocenter spacing of $2000 \dot{A}$. When illuminated by a well-collimated atom beam, this grating showed strong diffraction peaks at angles given by the familiar grating criterion. This stipulates that the portions of the atom's wave function that pass through adjacent slots in the grating traverse paths whose lengths differ by an integral number of wavelengths (so that constructive interference results). In this case the waves are the de Broglie waves of the atomis passing through the grating.

Several other phenomena demonstrated in the last few years also represent elements of atom optics. All these were based on the use of light to manipulate the atoms: the diffraction of atoms by a standing wave of light, the confinement of atoms in a single mode of a standing wave of light, and the reflection of atoms from the evanescent light wave that protrudes into the vacuum beyond a glass surface at which total internal reflection is occurring.

In view of such developments, one can envision interesting atom-optical systems containing several individual alements. Compound lenses whose iocal length is independent of the atom's speed are one example; a Fabry-Perot cavity for atoms is another. All atom optical elements will work better for slow atoms with their long de Broglie wavelength.

Recently a three-grating atom interferometer has been demonstrated. This opens up several possibilities, including precise studies of atomic properties, fundamental tests of quantum mechanics, and studies of phase shifts associated with atom-surface interactions. As rotation sensors, atom interferometers have a sensitivity ten orders of magnitude greater than that of optical gyroscopes with the same geometry. This means that they may ultimately permit much more precise inertial navigation and may possibly allow new measurements in geophysics and general relativity.

\section{CONTROLLING DYNAMICAL PATHWAYS}

The existence of a broad variety of intense coherent radiation sources permits the molecular scientist to prepare energy-rich systems with a high degree of specificity. The ability to use these preparations to methodically rearrange the 
molecular architecture in a chosen manner is a long standing goal that now is beginning to be realized. The tools required for this operaticit consist of both the radiation source and the ability to prepare the most meaningful reagents. In general, the uncertainty of unknown nuclear geometry can be avoided if extremely cold $(0.01$ to $10 \mathrm{~K}$ ) reagents are prepared. Some examples of current exciting research in which reagents are carefully tailored in preparation for laser-induced transformation are given below.

\section{Reactions with Aligned Reactants}

Molecular transformations are the basic actions in chemistry and biology. Thus, understanding and controlling them must be of primary importance. A major change in the study of elementary niolecular processes has occurred with the development of state-to-state molecular dynamics. This change has brought with it a very close interaction between theory and experiment. Of the critical parameters of the reactants and products of a reactive collision, control of the classical impact parameter has remained elusive. Recent developments will soon allow even this parameter to be effectively specified, as may be seen from the following specific example that has been realized.

The reaction of hydrogen atoms with neutral molecules is a fundamencal process that has attracted broad attention. Of the methods for obtaining hydrogen atoms, the photodissociation of binary hydrides is one of the more versatile in providing atoms with controllable translational energy. In the past, these atoms have been reacted with molecules at random orientations of collision. Thus reaction cross sections were averaged over impact parameter. In order to direct the $\mathrm{H}$ atom to a particular point in the neutral molecule, a complex of the hydride and the molecule is produced using the ultralow temperatures available in a supersonic jet. The hydride-bearing complex of known geometry is photodissociated with intense energy-specific laser radiation. The insertion of the $\mathrm{H}$ atom is at the point of attachment, while the energy of the projectile is chosen by the experimenter. The reaction-product state distribution is in general analyzed by quantum-state specific techniques such as laser-induced fluorescence.

The application of this technique to insert photodissociated tragments in a precise, geometrically and energy-controlled manner may aliow synthesis of new species in addition to de'crmining the orientation parameters of molecular transformations. The requirements of this promising field are the ability to synthesize molecular complexes with proper orientition of the photoactive subunit and the power is surgically dissociate the species.

\section{Preparation of Transient Configurations and the Transition State}

A revolution in state preparation and molecular spectroscopy has been produced by the application of frequency-selected multicolored laser pulse sequences. The most developed and productive of the techniques, stimulated emission pumping, has allowed preparation of vibrationally rich, energy-specific states. These states are being used to characterize and understand a wide variety of phenomena in molecular dynamics. An example of results already obtained is found in the precise location in energy and geometric configuration of the barrier for dissociation in the classic photochemical system, formaldehyde. This is in many ways the realization of the observation of the transition state.

The understanding of the consequences of anharmonic potentials in polyatomic systems is considerably more than the examination of simple tetraalomic molecules at high excitation energy for the display of chaotic motion. In this work, the time between pulses is variable and may be chosen to allow evolution of the intermediate state. The use of ultracold molecules combined with high-stability lasers allows preparing and probing the dynamical details of molecular systems of considerable coniplexity at a level of detail heretofore not imagined possible.

For over a half century the transition state has played a central role in the theory of molecular transformations. As the unstable saddle point between reactant and product, it has proven elusive for capture by the experimen- 
talist. There are a number of stable polyatomic negative ions whose neutral species are regarded as the transition state for simple processes such as hydrogen-atom exchange. The use of negatively charged molecular ions as a source for producing interesting neutral species by laser-induced photodetachment is well known. A considerable step forward has been made in the characterization of the transition state by the preparation and the laser spectroscopy of the photodissociation process of selected negative ions.

\section{Altering Dynamical Pathways by Intermolecular Interactions}

The control of dynamical pathways in molecular transformations is a long sought-after goal. The deveinprnent of the detailed state-tostate dynamics has provided a deep understanding of the nature of elementary molecular transformations. The extension of this methodology to the more complex systems generally encountered is sought in a step-by-step manner. It is now possible to alter the reactants in a seemingly minor way that results in profound dynamical consequences. The reaction of molecular chlorine with molecular bromine to produce chlorine bromide does not occur. However, the reaction of the dimer of molecular chlorine with molecular bromine to produce chlorine bromide occurs with virtually a gas kinetic cross section. In this manner the very weak bond of the chlorine dimer facilitates the breaking of the strong chemical bond of diatomic chlorine.

Weak bonds may stabilize a system with respect to breaking of strong bonds. The energy of interaction of argon and iodine is extremely small compared to the excitation energy. When the complex of argon and molecular iodine is irradiated at energies well beyond those required to dissociate the iodine molecule, emission from the iodine molecule is observed. By forming molecular complexes and using them in dynamical processes, the molecular scientist can begin to unravel the nature of molecular transformations in a medium.

\section{NONCLASSICAL STATES OF LIGHT}

The fundamental limit to all measurements or devices using light beams is the intrinsic noise of the electromagnetic field. Consider, for example, detection of a laser beam by a photodiode. Even at the relatively low power levels of a common HeNe laser, the limiting noise on the detected photocurrent is associated with the laser light. It is called shot noise on the basis of the idea that photons arrive randomly but must correctly be thought of as fluctuations in the amplitude of the laser light due to quantum-mechanical fluctuations in the vacuum state of the mode of the electromagnetic field filled by the laser beam. The state of the light field of a laser beam is a close approximation to perfect classical wave motion. For many experiments and devices, it is entirely adequate to think of light using this classical wave description. However, noise is important in an increasing number of situations, and this nonclassical quantum description is required to understand the basic fluctuations in the amplitude and phase of light. In order to study the nonclassical noise properties of light, there has been an explosion of activity during the last decade to produce new "nonclassical" states of light where the noisy quantum fluctuations can be altered experimentally. Obviously, this work has the potential of developing ways to reduce the noise in applications where light is employed.

\section{Squeezed States}

Large reductions in the quantum fluctuations of light were first achieved with "squeezed" light, for which the quantum fluctuations in the electric field are reduced below the vacuumfluctuation level during one portion of each cycle. The uncertainty principle requires that the variance of the field averaged over an entire cycle remain constant. This requirement means that squeezed noise reduction during one portion of the cycle results in a corresponding noise increase during the remainder of the cycle. This squeezing of the noise from one portion of the cycle to another has been accomplished by using parametric amplifiers based on a wide range of 
optical and microwave nonlinearities. Squeezed noise reductions of nearly an order of magnitude have been achieved using parametric downconversion in optical crystals. Parametric amplifiers based on four-wave mixing have also been demonstrated. In general, any losses in the generation or detection of squeezed light will degrade the level of squeezing. One of the major challenges at present is to understand the fundamental limits for these loss mechanisms. While minimizing the loss, the nonlinear mechanisms required for generating the squeezed light must be optimized. Very interesting questions remain about the basic limits of optical nonlinearities in a wide range of materials. Another interesting question for the future is the effect of the squeezed quantum noise on parametric oscillators above threshold in the regimes where bistabilities and chaos occur. Squeezed light has recently been observed for propagating solitons in optical fibers. Many other interesting regimes where squeezing can be important remain to be explored.

\section{Quantum Twinning}

Quantum twinning is a quantum noise phenomenon closely related to squeezing. A pump photon in a lossless nonlinear material can decay into two photons called the signal and idler photons. This quantum twinning process is at the heart of many of the nonclassical lightgeneration processes. Although the occurrence of the photon pairs can be random in time, any fluctuation in the signal beam will be exactly duplicated in the idler beam. Signal and idler beams can be separated into spatial or polarization channels and detected by separate photodetectors. Strong correlations have been measured between the signal and idler photon currents that can be entirely attributed to the quantum twinning process. These strong correlations allow high-precision spectroscopy and provide the opportunity to suppress fluctuations in the signal beam after detection of the idler beam.

\section{Number States}

Number states are ideal states of light where the number of photons detected in a given time interval is fixed. If the detected current from a number state were used to drive a loudspeaker, it would sound like machine gun fire, whereas laser light would sound like rain. Near-number states have been produced by constant-current pumping of diode lasers. If the efficiency for converting the pump electron into a photon in a given mode is nearly 100 percent, as it is for a laser well above threshold, then the constant electron rate converts directly to a constant photon rate. Laser noise suppressions of over an order of magnitude have been obtained by this mechanism. A future direction in this area is to build optical microstructures that emit into only one mode. Then emission excited by a constant-current source could result in a nearnumber state independent of lasing. These microstructures would also lead to very low threshold lasers. Another question is whether a photon number amplifier can be designed.

\section{Photonic Band Gaps}

Photonic band gaps are closely related to nonclassical radiation since an atom or exciton in a system where there are no photon modes cannot radiate. This can be thought of as eliminating the vacuum fluctuations from a particular region of space and frequency. Recently it has been found that a dielectric lattice with a diamond structure or even a lattice with asymmetric units can eliminate photon modes in all directions over a significant spectral range. Specific modes can be reintroduced into such a structure by controlled "defects." This is an exciting new direction in research, and there is sure to be much progress in this field. Lowthreshold microlasers, control of spontaneous emission noise, and nonclassical light generation will be of great interest in these new systems.

\section{Applications}

Nonclassical states of light have potential applications in a number of areas. Communications channels are ultimately limited in their 
data rate capacity by quantum noise. For example, a coherent communication system with a bit error rate of 1 in $10^{9}$ requires 9 photons for every bit of information. Squeezed light can reduce this to only 3 photons per bit. However, at present the limit due to more mundane noise sources is nearer to 30 photons per bit so that there is substantial room for improvement before the quantum limits are approached. Many lossless optical devires, e.g., optical switches, require a level of nonlinear optical activity that is exactly in the range where nonclassical light effects are important. This means not only that nonclassical light will be generated but also that the noise limits of these devices will be determined by yet-to-be-explored quantum processes.

Precision measurement is more likely to be the first application of nonclassical light. Optical interferometric measurements of phase can now be made where the limiting noise is well below the shot noise limit. In addition there are general schemes for quantum measurement called quantum nondemolition measurements. It is now possible to measure the amplitude of a light field without perturbing it. Of course the phase of the field will become noisier as a result of this measurement, but this is not a problem in many erperiments.

Methods have recently been suggested to build large-amplitude "macroscopic" states of jight that would be true quantum superposition states. For example, two states with different phases could be superimposed so that the system would be in both states until a measurement is made. Interference between these optical states could be easily demonstrated. These experiments would force the development of high-efficiency detectors required for generating the large-amplitude superpositions. These states are closely related to the ideas of quantum chaos and quantum computation. Studies of the macroscopic superpositions will clarify the physical conditions required for reversible quantum computation and whether there are systems that can be accurately described as quantum chaotic.

Finally, there continue to be a number of experiments and suggestions relating to the basic concepts of quantum mechanics in the field of nonclassical light. Before Bell passed away recently, he again questioned the true meaning of measurement in quantum theory. Is quantum measurement really well understood or do we have more to learn? Many of the experts can still disagree on the predictions of quantum measurement, whereas many others think that everything is cleared of mystery. Several experiments demonstrating Bell inequality relations are in progress and others have been suggested. Since this research deals with the essence of quantum paradox, it seems wise to keep trying what may at times seem obvious in the hope that some new insights will be obtained.

\section{TPAANS!ENT STATES OF ATOMIC SYSTEMS}

The key to understanding a vast array of complex atomic collision phenomena involving the transfer of energy, angular momenta, and charge is, in many important cases, the accurate description of the transient intermediate states of the collision complex. Improved experimental capabilities are making possible far more complete analyses of such states than has been heretofore possible, thereby permitting stringent tests of theoretical understanding. In addition, a host of novel physical processes are being studied experimentally for which theoretical understanding is only beginning to be developed. The scope of such processes encompasses all the interactions among photons, electrons, positive and negative ions, neutral atoms, and even antiparticles.

\section{Dynamics of Three-Body Systems}

While systems having two interacting particles may be described analytically, systems having three or more interacting particles can only be described approximately. Three-body systems are thus the prototypes of many-body systems. Experimental and theoretical progress in their description is a key to much of the physics of the everyday world. Indeed, the physics of many complex processes is governed 
by the interactions of three interacting particles (one or more of which is often a composite particle). A brief listing of some advances in the past year or two will serve to illustrate the intense activity occurring in this fundamental area.

High-resolution experimental measurements of the photodetachment cross sections for the negative hydrogen ion have uncovered recently an extremely rich spectrum of doubly excited states. Theorists meanwhile have uncovered unexpected propensity rules for optical transitions in three-body systems, which provide a good measure of understanding of these measurements. Among the basic issues addressed in these studies are the following: What is the nature of quantization in simple, few-body systems? What approximate constants of the motion govern that nature? What are the corresponding approximate quantum numbers? How good are they?

Recent experimental measurements of double photoionization of atoms have uncovered an intriguing empirical relationship of this process to electron impact ionization of the corresponding singly charged ions. The measurements have been so precise that they have also tested theoretical understanding of threshold laws for three-body breakup processes and, indeed, have led theory to predict alternative modes for threebody breakup processes applicable in different energy regions above threshold. Finally, the experimental measurements at high photon energies are providing severe tests of theoretical estimates of the ratio of double to single photoionization at asymptotically high photon energies.

Resonances in low-energy electron-atom collisions provide detailed information on transient states of negative ions. Many such states are doubly excited, and electron-correlation effects are of paramount importance in their theoretical description. Several years ago, experimental data on electron-Cs scattering were used to make a semiempirical prediction of a stable ${ }^{3} \mathrm{P}$ state of the negative $\mathrm{Cs}$ ion. The existence of this state has just recently been confirmed by $a b$ initio theoretical calculations.
Surprising as it may seem, certain very basic physical processes are only now being studied successfully. For example, theoretical calculations of electron impact excitation of atomic hydrogen have only recently achieved agreement with experimental measurements. In addition, with spin-dependent measurements of electron-hydrogen scattering providing guidance, theoretical agreement has also been achieved recently for the impact ionization channel over a wide range of energies, the threshold region being one notable exception, where new spin-asymmetry data have called attention to possible deviations from long-held theories of double escape. And experimental measurements of the angular and energy distributions of electrons produced in proton impact ionization of atomic hydrogen are just beginning to be made.

\section{Collisions at Ultralow Temperatures}

Technological progress in laser cooling and trapping of atoms over the past few years has permitted the experimental study of atomic collisions at millikelvin temperatures. Because the collision duration at such temperatures can be much longer than the natural lifetime of an excited state, spontaneous emission has a strong influence on excited-state collision processes. The low kinetic energies also cause collision processes to be extremely sensitive to perturbations by external electric, magnetic, and electromagnetic fields. This offers the prospect of controlling collision rates by external means.

Cold collision studies are not limited to atom traps. Laser velocity selection techniques have been used to study associative ionization of excited $\mathrm{Na}$ atoms at 60 millikelvin. Furthermore the development of cold atomic beam sources in the millikelvin energy range are a realistic prospect in the near future, thereby permitting the study of atomic collisions over a velocity range from high collision energies to the limit of quantum threshold behavior near absolute zero temperature. 
Highly Charged Ions

The technology for making and handling highly charged ions has taken a dramatic jump during the past few years, opening a whole host of important phenomena to study. The technological advances have taken several forms, including the electron-cyclotron resonance (ECR) and electron-beam ion sources (EBIS), the coming on line of heavy-ion storage rings, and the invention of the electron-beam ion trap (EBIT).

The modeling of nonequilibrium plasmassuch as those encountered in magnetically confined fusion devices, high-powered lasers, and stars-requires detailed knowledge of the cross sections for collisions of the various plasma components, including photons, electrons, atoms, and ions of various charge states. Experimental measurements for such collision processes are necessary to check the validity of theoretical models. As one example, measurements of electron-ion collision processes for highly charged ionic species in the atomic number range $15<Z<40$ are critically needed to further understanding of hot plasmas in fusion energy and $x$-ray laser research. Studies of the sensitivity of such processes to ever-present external fields are also urgently needed, particularly when dielectronic resonances are involved. For all of the collision processes relevant to nonequilibrium plasmas, the newly available sources of highly charged ionic species assure that both experimental and theoretical studies are ready to burgeon.

High-energy atomic physics continues to provide stringent tests of QED using one- and two-electron ions of high atomic number $Z$. Measurements of radiative decay rates in helium-like uranium produced in high-energy beamfoil experiments have already provided experimental results for the Lamb shift in U. Use of decelerated beams and of storage rings to cool the beams promises to greatly improve experimental accuracies. More recently, resonant laser studies of the $2 p-1 s$ transition in hydrogen-like ions near $Z=15$ have provided measurements of the Lamb shift. Future improvements in ion beam intensities as well as in laser intensities hold great promise for significant improvements in experimental accuracies.

The search for parity nonconservation in high- $Z$ hydrogenic ions is a promising means for high-energy atomic physics to test the standard model once intense enough ionic bearns are available. All atomic parity experiments to date have involved heavy atoms and are therefore limited by the requirement that the structure of these complicated atoms must be understood. Parity experiments in one-electron, high- $Z$ ions, where the structure is known precisely and where the high atomic number provides greater sensitivity to neutral currents, promise more stringent tests of the standard model.

\section{Supencritical Fields}

If there were a nucleus with supercritical charge $Z>173$, the 1 s atomic level would be bound by an energy greater than twice the electron rest energy. A K-shell vacancy in such a system would decay by spontaneous creation of an electron-positron pair, with the electron filling the vacancy and the positron being emitted. While no stable nuclei of such high $Z$ exist, supercritical atoms can be obtained as transient states in energetic ion-atom collisions, e.g., a high-energy uranium beam incident on uranium atoms might produce an "atom" with $\mathrm{Z}=184$. Such experiments with uranium have been carried out, and, instead of the expected continuous energy distribution of positrons, discrete positron peaks have been observed that are correlated with similar peaks in the electron coincidence spectrum. The origins of these peaks are currently unknown and constitute one of the lingering problems of physics.

\section{Particle-Surface Interactions}

Particle-surface interactions are of interest for a number of reasons. First, they may be used as probes of various characteristics of the surface. Second, they may be used to produce exotic atomic species. Third, they provide theory with the opportunity to develop novel approximation techniques appropriate to processes encompassing simultaneously vastly different time scales. As an example of the latter, consider fast-ion scattering on a surface at grazing incidence. The 
motion of the ion perpendicular to the surface may be described by near-adiabatic evslution, whereas that parallel to the surface rnay be described by sudden transitions characteristic of fast ion-atom collisions. Fourth, understanding mechanisms for ion neutralization at surfaces is important for modeling limiters and divertors in magnetic fusion devices.

As an example of particle-surface interactions as surface probes, consider the case of magnetic materials. The surface characteristics of magnetic materials may differ considerably from those of the bulk due to different electronic environments; e.g., a bulk paramagnet may have a ferromagnetic surface. Characterization of such surfaces is of great importance to magnetic recording technology. A promising new technique for probing surface magnetism is electroncapture spectroscopy utilizing, e.g., a 150-keV deuteron beam at grazing incidence to probe the topmost atomic surface layer. Characteristics of the surface are gleaned from charge state and electronic spin polarization of deuterons that strip electrons from the surface.

As an example of the use of particle-surface interactions to produce exotic atomic states, consider recent experimental observations of extremely rapid neutralization of slow, highly charged ions near a metal surface. The positively charged ions appear to attract large numbers of electrons from the surface into very highly excited atomic orbitals, thereby producing what has been dubbed a "hollow" or "superexcited" atom. The short time scale of $10^{-15} \mathrm{sec}$ for such neutralization is not understood theoretically as yet.

\section{NEW LIGHT GENERATION AND HANDLING \\ Optically Generated Beams of Sub-picosecond Pulses of THz Radiation}

Recently, there has been a great deal of work demonstrating the generation of $\mathrm{THz}$ radiation via excitation with ultrashort laser pulses. This frequency range is difficult to access by purely electronic means, and although single line $\mathrm{THz}$ (far-infrared) lasers do exist, essentially all previous spectroscopic investigations have used the far-infrared radiation from a mercury arc lamp. Scientifically this spectral region is important, containing the gap frequencies of superconductors, magnetic excitations, the far-infrared modes of lattices, and the rotational transitions of molecules.

Modern integrated circuit techniques have made possible the precise fabrication of micronsized dipoles, which, when photoconductively uriven by sub-picosecond laser pulses, can radiate well into the $\mathrm{THz}$ regime. An alternative and complementary approach has been to extend radiowave and microwave techniques into the $\mathrm{THz}$ regime through the use of antennas. Other sources based on various physical systems and effects include the emission of an electromagnetic shock wave due to a moving volume dipole distribution, i.e., electrooptic Cerenkov radiation, and the electromagnetic shock wave radiated by a propagating surfacedipole distribution. Most recently, radiation has been generated by photoconductively driving the surface field of semiconductors and of strained layer superlattices with ultrafast laser pulses.

Of these various approaches, the one most technically developed combines state-of-the-art microelectronic lithographic and processing techniques with high-speed electronics and ultrafast laser sources. Here, integrated-circuit Hertzian dipoles, optoelectronically driven by ultrashort laser pulses, are used to generate subpicosecond transients of electromagnetic radiation. The dipole structures fabricated on semiconductors have consisted of photoconductive gaps, microscopic dipolar antennas, and point sources produced by shorting coplanar transmission lines. If the ultrafast $\mathrm{THz}$ source is located at the focus of a spherical mirror or lens contacted to the back of the chip, a large fraction of the emitted radiation is captured and can be focused or collimated, providing nearly diffraction-limited imaging of the terahertz radiation. With these optoelectronic techniques, diffractionlimited beams of single-cycle terahertz electromagnetic pulses have been produced and measured, after propagation distances of several meters, with signal-to-noise ratios of better than 
10,000 and with time resolutions of better than 0.4 psec.

The coherent detection of these extremely broadband transients of electromagnetic radiation with subpicosecond time resolution makes this optoelectronic source an important new tool with many possible applications. The most immediate one is the spectroscopic characterization of materials in the terahertz (far-infrared) regime by time-domain spectroscopic techniques. Here, the measured input and propagated electrical pulses are Fourier analyzed, and because the actual electrical field is measured, both the amplitude and phase of the Fourier components are obtained. Consequently, the frequency-dependent absorption and dispersion can be determined for any far-infrared transmitting material. This technique has some powerful advantages in producing results compared to those of traditional continuous-wave spectroscopy. First, the detection of the far-infrared radiation is extremely sensitive. In terms of average power, the sensitivity is more than 1000 times that of liquid-helium-cooled bolometers. Second, because of the gated and coinerent detection, the thermal background that plagues traditional measurements in this frequency range is observationally absent.

Using the time-domain spectroscopy method, researchers have characterized the effects of the strong lattice resonance at $6.3 \mathrm{THz}$ of lithium tantalate; zone-refined ultrahigh-purity silicon has been shown to be almost transparent and dispersion free from low frequencies to beyond $3 \mathrm{THz}$ and thereby to be an essentially perfect material for $\mathrm{THz}$ optical components; the mobilities and carrier densities of device-grade silicon wafers have been determined; the strengths of the water vapor lines from 0.2 to $1.45 \mathrm{THz}$ were measured with the best precision to date. In contradiction with early highly publicized claims for high-temperature superconducting (high- $T_{c}$ ) transmission lines, the high. $T_{c}$ substrates yttriastabilized zirconia (YSZ) and lanthanum aluminate were shown to be incapable of supporting high-bandwidth transmission on high-T, lines, due to the large $\mathrm{THz}$ absorptions measured in these materials.
Another important area of application of these pulsed optoelectronic $\mathrm{THz}$ sources is timedependent spectral characterization after an lititiating event, made feasible by the synchronization between the ultrashort light pulses and the THz pulses. An important measurnment in this area was the time-dependent rise of the mobility of GaAs from a small initial value to approximately the static value in a few picoseconds after the essentially instantaneous creation of the photocarriers.

A completely different type of application would be ranging measurements with a possible precision of better than 100 microns. For example, it should be possible to characterize plasmas with exceptional spatial resolution. Moving interfaces can be followed during processing in hostile environments. Arrays of sources and detectors can be used, and results have already been reported. The transmission and detection of images are real possibilities. The fact that signals of tens of millivolts are obtained at receivers allows for possible remote triggering of devices. Finally, it is clear that these beams have tremendous capacity as a communications channel.

\section{Second Harmonic Generation in Guided Wave Structures}

Second harmonic generation (SHG) is an area that has sustained scientific and technical interest due both to its fundamental nature and to its large applications potential. On the one hand, harmonic generation in channel and fiber waveguides has been refined to the point that semiconductor diode lasers can now be efficiently doubled to produce more than adequate amounts of blue light for data storage applications. On the other hand, why harmonic generation occurs in glass fibers after an appropriate preparation by radiation with light beams continues to puzzle physicists. Similar comments could be made about solitons in fibers, whose physics has turned out to be engrossing; and which ure being considered for prototype long. haul communications systems. The same mechanisms have allowed compression of pulses 
down to 6 femtoseconds in duration, only a few optical cyries.

Waveguide geometries are ideal for nonlinear optics because the propagating electromagnetic fields can be confined to cross-sectional dimensions of the order of their wavelength, for distances limited only by material absorption or scattering. In the best integrated optics waveguides, centimeter path lengths are possible, whereas optical fibers allow interaction distances of meters to kilometers. The combination of strong confinement and diffractionless propagation over long distances is critical, since the efficiency of most interactions depends on both intensity and interaction distance.

One of the first nonlinear interactions to be studied in waveguides was second harmonic generation. In the latter half of the past decade, it was realized that the doubling of the 50- to $100-\mathrm{mW}$ output of semiconductor lasers to produce a few milliwatts of blue light was possible and was sufficient for optical data storage applications. Subsequent rapid progress was owing to key developments in the areas of materials and new phase-matching techniques. In the early 1980s, the largest useful nonlinear coefficient available for phase matching in channel waveguides was that of lithium niobate. Now, new organic materials have nonlinearities more than 10 times larger than that of lithium niobate. Some of these organic materials have been grown in crystal form and even in waveguide geometries. Since the optimum conversion efficiency varies as the square of the coefficient, this increase in nonlinearity corresponds to orders-of-magnitude improvement in conversion efficiency.

Approaches to solve the phase-matching problem are waveguide versions of the old idea of stacking nonlinear platelets, one-half ccherence length thick, with successive platelets rotated by 180 degrees to periodically reverse the nonlinearity direction. In lithium niobate, this was achieved by reversing the polarity of the adjacent domains along the c-axis of lithium niobate. For this case conversion efficiencies of as much as 30 percent per watt per square centimeter have been demonstrated, and 10 times that is projected. Another interesting possibility, in the early stages of development, is the use of poled polymers with alternate regions poled along opposite directions. In ionexchanged potassium-titanyl-phosphate waveguides, within one coherence length, it is possible to have one region in which the phase mismatch is positive and one in which it is negative. If it is ensured that the total phase mismatch is zero over one coherence length, very efficient conversion occurs.

Given the vigorous and continuing effort to develop single-crystal noncentrosymmetric media, it came as a great surprise that glass fibers containing dopants such as $\mathrm{GeO}_{2}$ were changed into SHG-active media when subjected to intense $1060-\mathrm{nm}$ radiation. The second harmonic signal grew over many hours of exposure. Soon thereafter, it was discovered that "seeding" at the outset with green light reduced the preparation time to minutes, and that a grating had been formed inside the fiber to produce phase matching. To date, it has been established that multiphoton absorption effects are important, that the tensor properties of the nonlinearity are those associated with the product of the third-order susceptibility and a large direct-current field of the order of 100,000 volts per centimeter, and that the SHG signal can be "bleached out" with green or infrared radiation. Many fascinating features of this process have been uncovered, but the basic physics remains elusive.

\section{Coherent XUV Radiation from Strong-Field Multiphoton Processes}

The advent of high-power, short-pulse optical lasers has opened a new regime for the study of laser-atom interactions. Experimental and theoretical studies of atoms in strong laser fields have already yielded a host of new phenomena, some of which may have technological applications. One particular example is the generation of coherent extreme-ultraviolet (XUV) radiation by high-order harmonic conversion in atomic gases. The results of recent research suggest that it should be possible to develop a tabletop, tunable source of coherent XUV radiation using 
a subpicosecond, 10- to 50-GW, optical dye laser as the pump. Theoretical calculations predict that peak output powers in excess of $1 \mathrm{~kW}$ in the 5- to $40-\mathrm{eV}(30-$ to $250-\mathrm{nm})$ range should be possible. If realized in the laboratory, the peak power of such an XUV source would exceed by several orders of magnitude what will be produced by the advanced synchrotrons now under construction. A tabletop XUV source would find broad application for some problems in material sciences, chemistry, molecular biology, and AMO sciences, where high peak power, short pulse $(<1 \mathrm{psec})$, and narrow bandwidth are essential.

\section{Ultrafast X-ray Pulses from Laser-Produced Plasmas}

A new important class of time-resolved measurements in plasma and solid-state physics will become possible using ultrashort pulses at $x$-ray wavelengths. The availability of picosecond $x$-ray pulses will have significant impact in areas such as time-resolved $x$-ray diffraction and extended $x$-ray absorption fine structure (EXAFS) and will provide an ideal source for calibrating time-resolved $x$-ray detectors such as streak cameras and framing cameras. Such uitrafast $x$-ray sources could be used to perform time-resolved $x$-ray scattering of rapidly evolving materials (such as diffraction from structures undergoing rapid phase transitions), time-resolved photoemission, and flashlamp pumping of $x$-ray lasers.

Recently, such ultrafast $x$-ray sources have been demonstrated. In these laser-driven $x$-ray sources, a high-temperature plasma is created when an intense laser pulse is focused onto the surface of a solid. An ultrafast pulse of $x$-ray radiation is emitted from such a plasma when the laser pulse duration is less than a picosecond. $X$ rays from these sources are incoherent but have high brightness as a result of the small source size, short lifetime, and high temperature of the radiating plasma. X-ray pulses with a duration of a picosecond or less have been observed. These pulses are more than an order of magnitude shorter than those produced by any other $\mathrm{x}$-ray source.
Demonstration of short-pulse $x$-ray emission required the development of two experimental tools. First, a high-power, ultrashort pulse laser system with low prepulse energy was needed as an excitation source. Low prepulse energy or, equivalently, a sharp leading temporal edge, is essential, because energy deposited in advance of the main pulse can ablate target material and form an opaque vapor that will absorb the laser energy in front of the solid surface. Second, an $x$-ray detector with picosecond time resolution was required.

The laser pumped $x$-ray source can be described as follows. When a solid is illuminated by intense laser light, electrons in the material absorb energy and are rapidly heated. Hot electrons can subsequently ionize the much cooler atoms, forming an x-ray-emitting hightemperature plasma spark at the surface of the solid. Deposition of laser energy at high densities is crucial for the production of short $x$-ray pulses. Electron heating and the rise time of the emitted $x$-ray pulse will be comparable to the laser pulse duration. Short $x$-ray pulses are therefore expected if the plasma can cool rapidly. Deposition of laser energy at solid density allows rapid cooling because (1) the associated high thermal gradient allows rapid conduction of electron energy into the bulk of the solid beyond the optical skin depth; (2) the associated high pressure gradient allows rapid cooling by expansion into the vacuum in front of the surface; (3) nonequilibrium conditions in the underionized plasma allow hot electrons to cool rapidly by inelastic collisions with atoms and ions.

Scaling laws predict that brighter and more efficient $x$-ray sources will be obtained by the use of more intense laser pulses. Laser systems capable of generating $100-\mathrm{fsec}$ pulses with energies of several joules now appear to be possible, and such lasers are under development at several laboratories. Given the predicted scaling law for $x$-ray conversion efficiency, the xray yield from plasmas generated by such laser sources should exceed 10 percent at an absorbed irradiance in excess of $10^{18} \mathrm{~W} / \mathrm{cm}^{2}$. Consequently, the next generation of laser-produced plasma 
$x$-ray sources will yield extremely short-duration, high-intensity, and short-wavelength pulses. Such sources will increase the time resolution in experiments involving traditional $x$-ray scattering techniques. Fast $x$-ray streak cameras will find application by themselves for high time resolution in experiments that use relatively long-duration $x$-ray sources such as synchrotrons. In addition, the high-density plasmas produced under sub-picosecond illumination can be used to test our understanding of the physics of highly excited solids.

In summary, the ultrashort, intense $x$-ray pulse represents a source with many unique and useful features for applications in time-resolved measurements. The intrinsic synchronization of the $x$-ray pulse with the short optical pulse is ideally suited to pump-probe techniques and allows for low-jitter triggering of associated electronics such as gated detectors. Furthermore, the $x$-ray source is broadband in the sense that a wide range of energies can be accessed by the proper choice of target material. Specific applications for such a source include timeresolved $x$-ray diffraction and EXAFS in order to study dynamical changes in, respectively, the long-range and short-range order of condensed matter. Another important application is flash radiography, in which a dynamic system can be backlit by the ultrashort $x$-ray pulse to obtain an image of the instantaneous absorption profile.

\section{CLUSTERS}

One of the most active and fertile new areas to emerge in atomic and molecular physics over the past decade has been the study of small aggregates of atoms-clusters-both in the condensed phase and in beams. Particularly with the advent in the early 1980s of laser-vaporization techniques for the production of supersonic beams of these species, a wide range of fascinating and potentially very significant new science has been opened for study. Using these techniques it is now quite straightforward to produce intense beams of clusters in the size range from two to several thousand atoms of virtually any element or combination of elements in the periodic table. While these techniques are a direct outgrowth of decades of developments in the atomic, molecular, and laser physics communities, the emerging field of clusters is likely to have its largest impact in chemistry and materials science. In fact it should provide one of the most direct and effective bridges between the AMO sciences and these more applied disciplines. Three examples of this very broad and rapidly moving research are: asc $=:$ mmarized below, beginning with the most famous of all clusters, $\mathrm{C}_{60}$.

\section{"Buckminsterfullerene"}

In the midst of the first supersonic cluster beam experiments on carbon in 1984-1985, a bizarre distribution of even-numbered clusters was discovered in which the "magic" numbers of 60 and 70 appeared particularly abundant and stable. Following an analogy with the geodesic dome architecture of Buckminster Fuller, the structure of these clusters was proposed to be made up of a network of 5- and 6-member rings curved around to form a closed, hollow shell. In the case of $\mathrm{C}_{60}$, this structure would constitute a perfect molecilar soccer ball, whereas in the case of $C_{70}$ the shape would be better described as a rugby ball. Experiments in the supersonic beams and on the clusters levitated in a magnetic trap strongly supported this remarkable structural proposal and provided evidence that the entire series of even-numbered clusters from 32 to well over 600 atoms in size were also "fullerenes"-hollow carbon cages built out of a hexagonal graphitic sheet that closed on itself through the inclusion of 12 pentagons. Strong evidence was also found for the spontaneous formation of a variety of metallofullerenes where a single metal atom was caught within the hollow cage.

In 1990 the correctness of these structural proposals was stunningly verified by the isolation of the first macroscopic samples of $C_{60}$ and $\mathrm{C}_{70}$ from carbon soots condensed in an atmosphere of helium. The method of generation was soon imiproved through the use of a simple carbon arc design that should be readily scaled to the production of large (ton) quantities at low cost. The result is that a third form of pure 
carbon is now available for the first time. In addition to diamond and graphite, there are now fullerenes like $\mathrm{C}_{60}$.

While the full significance of this discovery of fullerenes may rot be clear for many years, it is clear that a huge new area of research has been opened, much of which will be in chemistry and materials. From the viewpoint of AMO sciences there are a host of fascinating topics to pursue. One is the effort to fully understand the process under which these fullerenes form with high yield in condensing carbon vapors and plasmas. The current best evidence suggests the critical aspect is balancing the rate of graphitic sheet growth with the rate of annealing of these sheets to tie up darigling bonds (through the inclusion of pentagons). Is this correct? How efficient can it be made? Can conditions be found where metal atoms or ions are efficiently trapped inside? Experiments with levitated clusters made by laser vaporization have shown that fullerenes such as $\mathrm{C}_{60} \mathrm{La}$ and $\mathrm{C}_{60} \mathrm{U}$ are readily produced. There is speculation that the mechanism for formation involves capture of the positive metal ion in a curving graphitic sheet. If the mechanism of capture can be linderstood, it may be possible to devise a large-scale production strategy, perhaps involving laser innization of metals within the critical region of a condensing carbon vapor. The resulting metallofullerenes will provide fascinating examples of perturbed a.oms in a high-symmetry cage and could have major technological significance. The cage in $\mathrm{C}_{60}$ is large enough to contain any atom in the periodic table. By varying the atomic number of the atom inside, it will be possible to systematically tune the optical, chemical, and redox properties of the carbon cage.

In combustion science, one of the longeststanding problems has been the process of soot formation. It has been suggested that the same mechanism that leads to the curving of growing graphitic sheets to form $\mathrm{C}_{60}$ may also lead in the presence of hydrogen to the formation of spiraling, nautilus-like structures that may be the critical nuclel for the formation of soot in flames. The 1987 discovery of $C_{60}$ and the other fuller- enes in sooting flames provides support for this notion. Still, the issue is far from clear, and extensive further research with the help of the AMO community appears well justified.

\section{Semiconductor Clusters}

Clusters of semiconductors like sillicon have been studied extensively over the past few years with the new techniques. Even though it is the closest neighbor to carbon in the periodic table, $\mathrm{Si}_{60}$ is nothing like $\mathrm{C}_{60}$ and the other fullerenes. Unable to form strong double bonds, silicon is not able to satisfy its bonding needs in a twodimensional graphite-type lattice. Like most elements, pure silicon can only fulfill its bonding need in a full three-dimensional lattice, producing a bulk solid. While the physics and chemistry of bulk solids constitute a well-developed field with a strong, predictive theoretical underpinning, the problem of what is going on with the physics and chemistry of the surface is far more difficult. This is true as well with the surface of the $\mathrm{Si}_{60}$ cluster. Here the dangling bonds that would otherwise connect to other silicon atoms in the bulk solid now dominate the surface chemistry and physics. Unlike $\mathrm{C}_{60}$, which in the "buckminsterfullerene" structure is a true molecule, $\mathrm{Si}_{60}$ is better thought of as a piece of the bulk solid where the surface has been reconstructed as much as possible. And like the surface of crystalline silicon, it is the precise way this reconstruction and partial tying up of the dangling bonds on the surface of the $\mathrm{Si}_{60}$ cluster that determines its physics and chemistry.

One of the birthrights of the new cluster science is the ability to systematically vary the cluster-to vary the number of atoms in the cluster, its charge, the number of chemisorbed atoms on the surface, and at least in some cases its geometrical structure. This allows clusters to be excellent testing grounds for the predictions of new fundamental theories as to the nature of surface reconstruction on semiconductor surfaces and the effect this has on surface chemistry. As with carbon where 60 is a "magic" number, recent research is turning up a new set of magic numbers in silicon and germanium 
clusters. These special clusters (those with 21, $25,33,39$, or 45 atoms) appear to have adopted particularly stable structures that are considerably less reactive than those of their neighbors. This observation in turn has stimulated a flurry of calculations to understand the nature of these special structures and to offer predictions that can be tested by further cluster experiments.

For silicon, germanium, and gallium arsenide, there are indications that the current generation of supercomputers will soon be able to calculate directly and accurately at least the ground-state structure for clusters as large as $\mathbf{5 0}$ to 100 atoms using the new techniques of simulated annealing combined with density functional theory. This is likely to be an area of the AMO sciences that will attract major interest over the next decade.

\section{Metal Clusters}

Far more complicated than the surface restructuring of a cluster of a network-bonded covalent solid like silicon is the problem of metal surfaces. Here too the new cluster beam science has shown major vitality. Although the results are ofien much more complicated (and in many cases are still inexplicable at our current level of understanding), some beautifully simple pictures have emerged. One of the most striking is the extent to which a simple particle-in-a-sphericalbox quantum-mechanical shell theory appears to describe the electronic structure of a wide range of metal clusters. For those metals whose bulk electronic band structure is fairly well described near the Fermi energy as a free-electron metal, one might guess that a small ball of the metal still has its electrons traveling freely but forced to reflect off the inside of the spherical surface of the ball. In fact, with small variations, this turns out to be a remarkably accurate picture, even for metal balls made up of only a few dozen atoms. For metals such as sodium, copper, or gold, this spherical box theory predicts that clusters of certain magic number sizes (e.8., 2, 8, 20, 40, 58, and so on) should be particularly stable. The theory is formally the same as the shell model of the nucleus. Much of the metal-cluster research over the past few years has focused increasingly on determining the degree to which this simple model applies not only to static properties such as structure and stability, but also to time-dependent responses such as absorption and light scattering. In extending this approach to clusters in the size range of several thousands, we will learn in detail how the metallic state develops.

One of the most challenging frontiers in metal cluster research is the understanding of the physics and chemistry of transition metal clusters. Research thus far has verified that the same sorts of complex dissociative chemisorption phenomena that dominate the catalytic properties of bulk transition metal surfaces occur also on clusters with just a few atoms. Here the simple free-electron shell model theories no longer work. But the cluster experiments are still often extremely intriguing. For example, it has been found that clusters of cobalt switch from being completely inert toward hydrogen, to effectively dissociating this molecule with unit sticking probability, simply by increasing the size from 9 to 10 atoms. Two geometrical isomers of the nineteenth cluster of niobium have been found to have completely different chemistries. Although there is as yet no firm understanding of these results, there is much activity by electronic-structure theorists to begin to grapple with this problem.

There is little doubt that such activity both on the experimental and theoretical sides of the AMO sciences will be a major challenge for many years to come. While further discoveries on the order of $\mathrm{C}_{60}$ may be rare, it does seem likely that research on clusters will remain one of the most vital and productive areas of physics.

\section{III Atomic Physics at User Facilities}

User facilities provide both diverse and unique opportunities for research in atomic physics. The beams of energetic particles and photons delivered by present-day particle accelerators and synchrotrons are used to probe and 
to manipulate the structure of atoms and molecules and to study their dynamical interactions. Such facilities permit the realization of extremely high transient fields in atoms and molecules. Even though the forces between atomic particles are well known, the prediction of such dynamical interactions requires the solution of the timedependent many-body problem, which still remains a major theoretical challenge. The classes of interactions that are studied at such facilities also play key roles in many other areas of science, such as nuclear, solid-state, and plasma physics, biology, chemistry, medicine, and astrophysics. Below are selected examples of the roles of three types of user facilities in atomic and molecular sciences.

\section{SYNCHROTRON RADIATION FACILITIES}

The unique properties of electromagnetic radiation produced by fast electrons in synchrotron rings make it ideal for atomic and molecular physics research. The implementation of advanced "wiggler" and "undulator" technologies now makes such devices capable of delivering intense, monochromatic, polarized, pulsed beams of photons at wavelengths ranging continuously from the infrared to the hard $x$-ray region. The enhanced intensity makes gasphase experiments possible. With several major new facilities either operating or soon to come on line in the United States, exciting scientific opportunities abound. The production of deep core-excited states of complex atoms by photoionization and their subsequent decay will severely test our understanding of the effects of electron-correlation and relativity on atomic structure. High-resolution studies of the decay dynamics of selectively excited molecular states will provide new insights into the fundamental mechanisms of molecular physics. The coupling of lasers with synchrotron radiation in so-called two-color experiments will open up a new experimental frontier for the study of excited molecular states and dynamical interactions using pulse-probe techniques.

\section{PARTICLE ACCELERATOR FACILITIES}

The field of accelerator-based atomic physics has developed and prospered during the past three decades at facilities primarily (or formerly) designated for nuclear physics research. The continuing vitality of this field is evidenced by the development of dedicated new facilities for experimental atomic physics. With the addition of multicharged ion sources and radio-frequency boosters, accelerator facilities can now deliver beams of ions of nearly all elements over a wide range of charge states, and with kinetic energies ranging from a few kilovolts to hundreds of gigavolts. Highly excited atomic states created in interactions of energetic ions with gaseous and solid targets permit detailed probing of electronic structure and dynamical interactions over a wide range of atomic parameters. The level of sophistication of experiments has fully kept pace with accelerator development. Scientific objectives range from structural and dynamical effects, such as electron correlation, to the most fundamental and definitive tests of quantum electrodynamics. Phenomena such as ion channeling in crystals offer alternative ways to probe fundamental electron-ion interactions. "Coulomv explosions" produced by energeticion impact on molecules are providing new insights into molecular dynamics and structure. Fast, highly charged "hammer" ion beams from heavy-ion accelerators are also directed through gas target cells to generate very-low-energy, highly charged recoil ion beams for further collision studies.

Energetic collisions of ions and targets of high atomic number may permit the transient achievement of supercritical fields, in which excited atomic states decay by emission of electron-positron pairs. As ion velocities approach the speed of light, atomic collisions may be viewed as virtual photon-photon collisions because of the Lorentz contraction of the nuclear Coulomb field. In effect, highly charged ions behave like an intense flux of high-energy gamma rays, providing new opportunities and challenges to both experimenters and theorists.

\section{HEAVY-ION STORAGE-RING FACILITIES}

The application of circular accelerator technology to the storage of heavy-ion beams has been made possible by development of effective ion-beam cooling techniques. The dramatically 
enhanced ion-beam luminosity due to recirculation opens up exciting possibilities for atomic physics research. Heavy-ion storage-ring facilities are now operational in Europe and Japan and have already proven their raw power as atomic physics research tools. High-resolution measurements using the rings' own electron cooler beams are providing long-awaited tests of the theory of dielectronic and radiative recombination in electron-ion collisions. The laser and electron cooling of charged particles aliso offers a unique opportunity to study phase iransitions in a confined one-component plasma. It may be possible to probe this "crystallization" of stored ion beams by laser scattering. In acidition to revolutionizing the study of electronion collisions, storage-ring facilities will open up many other new opportunities for research using techniques developed in accelerator-based atomic physics for ion-atom and ion-solid interactions.

\section{IV \\ Impacts of AMO Sciences on Modern Technologies}

As the preceding sections of this research briefing have shown, developments originating in atomic, molecular, and optical sciences now enter intimately into modern technologies, and it is useful to review some of these here to emphasize the synergism.

Understanding and exploiting the stimulated emission of electromagnetic radiation by molecules and atoms led AMO scientists to invent the maser nearly four decades ago and subsequently to invent the laser. The impact of lasers in their many forms on basic sciences and in enabling development of a host of commercial and military technologies has been nothing short of staggering. Lasers are now used routinely to weld materials ranging from retinas to inetal plates. Laser-guided munitions were important constituents of the arsenals put to heavy use in 1991. Starting with optically pumped liquid-dye lasers, now augmented by much smaller and cheaper solid-state diode lasers, frequency- tunable lasers have revolutionized optical spectroscopy. Examples range from coherent antiStokes Raman spectroscopy (CARS), important for modern combustion research, to remote detection and analysis of trace compounds for environmental research and monitoring, to use of femtosecond pulses of laser light to monitor (perhaps even to control) chemical reactions as they actually occur. The last is stimulating growth of a new field called laser femtochemistry. Tunable lasers permit the detection and mechanical control-cooling to microkelvin temperatures and trapping-of individual atoms and ions. It has even been demonstrated that such laser light forces may be used as "optical tweezers" to manipulate living microorganisms (in solution), objects inside living cells, or even individual biomolecules such as DNA.

The focal point of "photonics" and modern optics is the laser. Ring-laser gyroscopes are widely used in both the military and civilian sectors. Lasers coupled with-or even part of-low-loss optical fibers are enabling wider bandwidths and more secure communications. Optical amplifiers in active fibers are being used in the next undersea cable in the Pacific Ocean. Possible use of soliton lasers in long-haul communications is now being evaluated seriously. The growing impact of optics on computing may move from peripherals-laser printers, scanners, two-dimensional data storage on optical disks, and perhaps even three-dimensional holographic data storage in the future-to use of optical circuits in central processors and memory.

AMO scientists have invented a rich variety of spectroscopic techniques based on resonant absorption or emission of electromagnetic radiation. Early examples include nuclear magnetic resonance (NMR) and electron spin resonance (ESR), with medical imaging devices being a well-known application of the former. Atomic clocks are the core of several technologies, a noteworthy example being the rubidium atomic clock aboard each earth satellite in the Global Positioning System (GPS). Atomic magnetometers based on optical pumping techniques are used in applications ranging from geophysical studies, and oil and mineral prospecting, to 
remote detection of submarines. Even more accurate clocks and sensitive magnetometers are now being developed using laser-based techniques. Future advances will use slowed or trapped atoms or ions.

The design of modern arc lighting involves determining the interaction potentials between various atomic species and modeling the output for various constituent concentrations and iemperatures. Spectral control for specific applications can thereby be achieved, along with optimum operating energy efficiency.

For materials science applications, careful control of neutral thermal beams contributes to the growth of new materials by molecular-beam epitaxy (MBE), and basic atomic and molecular collision processes in the gas phase and with surfaces are the key to understanding and further development of reactive-ion etching techniques. Diffraction-limited projection of circuit mask images onto VLSI chip wafers with multi-element classical optics in the visible portion of the spectrum will give way to even higher circuit densities with use of ultraviolet and soft $x$-ray light sources and optical components. These technologies are based on beams of atoms and molecules generated using the techniques of the AMO sciences and depend on atomic and molecular collision processes. 

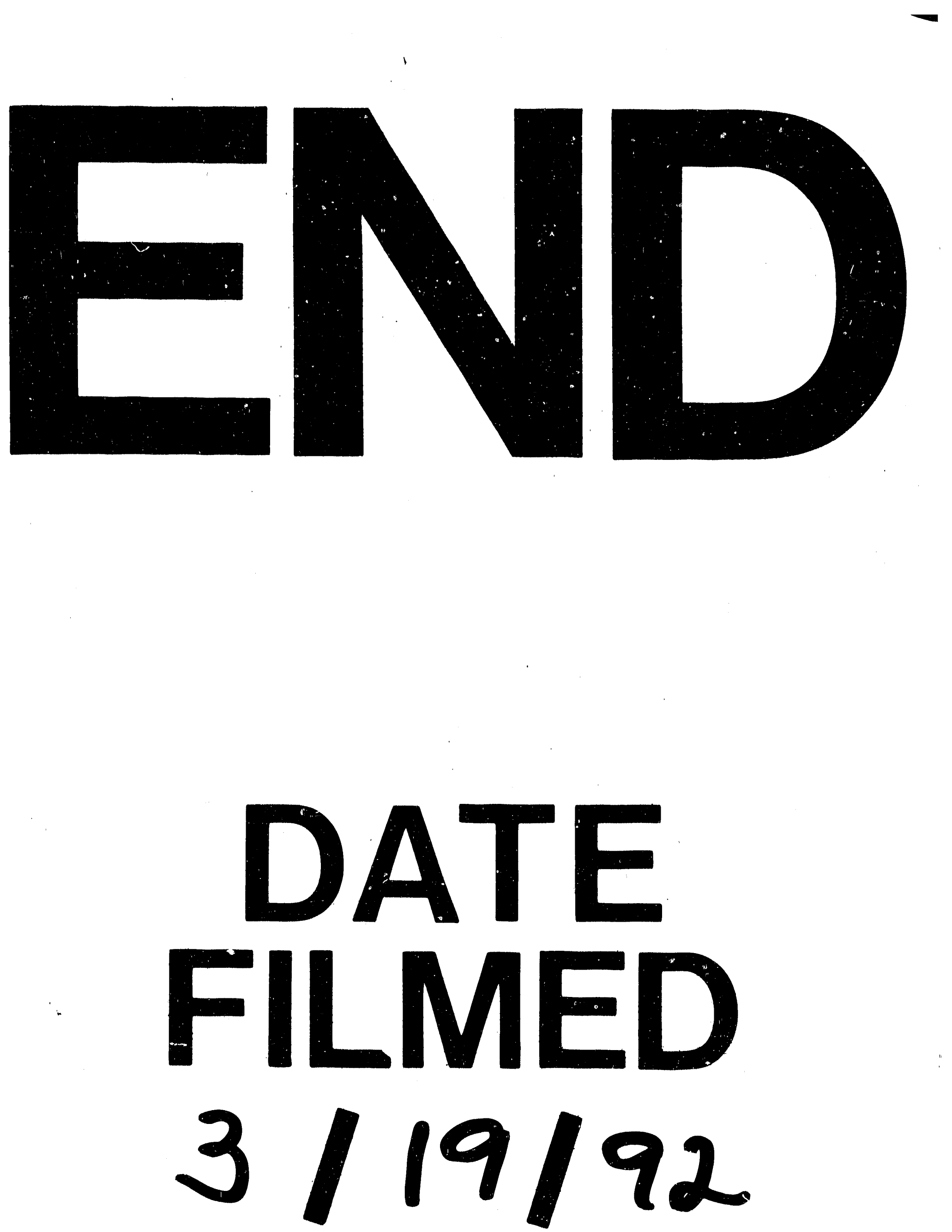
